Mon. Not. R. Astron. Soc. 000, 1-9 (2002) Printed 31 October $2018 \quad$ (MN LATEX style file v2.2)

\title{
An analysis of the emission line spectra of AG Pegasi between phases 7.34 and 9.44
}

\author{
Marcella Contini* \\ School of Physics and Astronomy, Tel Aviv University, Tel Aviv 69978, Israel
}

Accepted . Received ; in original form

\begin{abstract}
The UV and optical spectra from the HST FOS and from the FAST spectrograph at the Whipple Observatory reported by Kenyon et al (2001) at different phases are analysed, leading to new results about the configuration of AG Peg. The FAST spectra contain both $\mathrm{H} \alpha$ and $\mathrm{H} \beta$ lines, whose ratio changes with phase indicating that different nebulae contribute to each spectrum. In particular, the spectrum emitted from one of the nebulae must be collision dominated, in order to justify the relatively high $\mathrm{H} \alpha / \mathrm{H} \beta$. Consistent modelling by the code SUMA, which accounts for the coupled effect of the photoionisation from the hot star and the shock, shows the important role of at least three nebulae: 1) the nebula between the stars, downstream of the shock created by collision of the winds, which propagates in reverse towards the WD, 2) the nebula downstream of the shock expanding in the outskirts of the giant atmosphere, and 3) the shock propagating outwards the binary system, that is not reached by the hot source radiation. The relative contribution of the three nebulae to the final spectra at different phases are calculated for all the lines. It is found that the contribution to the different lines changes with the viewing angle of the system. The relatively low preshock density and magnetic field adopted in the modelling of the expanding shocks reveal that they are merging with the ISM, thus explaining the slow decline of AG Peg.
\end{abstract}

Key words: shock waves;stars: binaries: symbiotic-stars:individual: AG Peg

\section{INTRODUCTION}

AG Pegasi (AG Peg) is a symbiotic binary consisting of a white dwarf (WD) and a red giant (spectral type M3). A continuing slow decline of the far-UV and near-UV continuum is noticed by Kenyon, Proga, \& Keyes (2001). They claim that the eruption of the hot component in AG Peg may be the slowest classical nova outburst ever recorded. AG Peg evolution was followed by the observations (Kenyon et al 1993, Kenyon et al. 2001) and modeled by the interpretation of the line and continuum spectra (e.g. Contini 1997).

The collision of the winds from the hot star and the red giant in symbiotic binaries creates a complex hydrodynamic structure (Nussbaumer 2000 and references therein). The emitting gas within the system is then ionised and heated both by the photoionising flux from the hot star and by shocks. In a previous paper (Contini 1997, hereafter Paper I) the observed continuum and line spectra from AG Peg at phases earlier than $\phi=7.12$, have been explained by

\footnotetext{
* E-mail: contini@ccsg.tau.ac.il
}

composite models accounting both for photoionisation and shocks. Indeed, the complex structure of the system requires schematic models which represent roughly the real picture. The consistency of calculation results for the line spectra in the UV and in the optical ranges, as well as for the spectral energy distribution (SED) of the continuum leads to a better understanding of the physical characteristics of the system.

Following the results of Paper I, two shock fronts are created at collision of the winds between the stars, one propagating in reverse toward the WD and the other propagating outwards the system. Moreover, recent observations of symbiotic novae show that spectra are also emitted from downstream of shocks propagating outside the system and eventually merging with the ISM (Contini \& Formiggini 2001). At phase 7.05 of AG Peg the expanding shock has already passed over the red giant and slightly accelerates propagating in the atmosphere of the red giant, opposite to the WD, where the density gradient is decreasing.

New observations are presented by Kenyon et al. (2001, hereafter KPK01). They provide optical and UV spectra at phases 7.34 - 9.44, acquired with Faint Object Spectrograph 


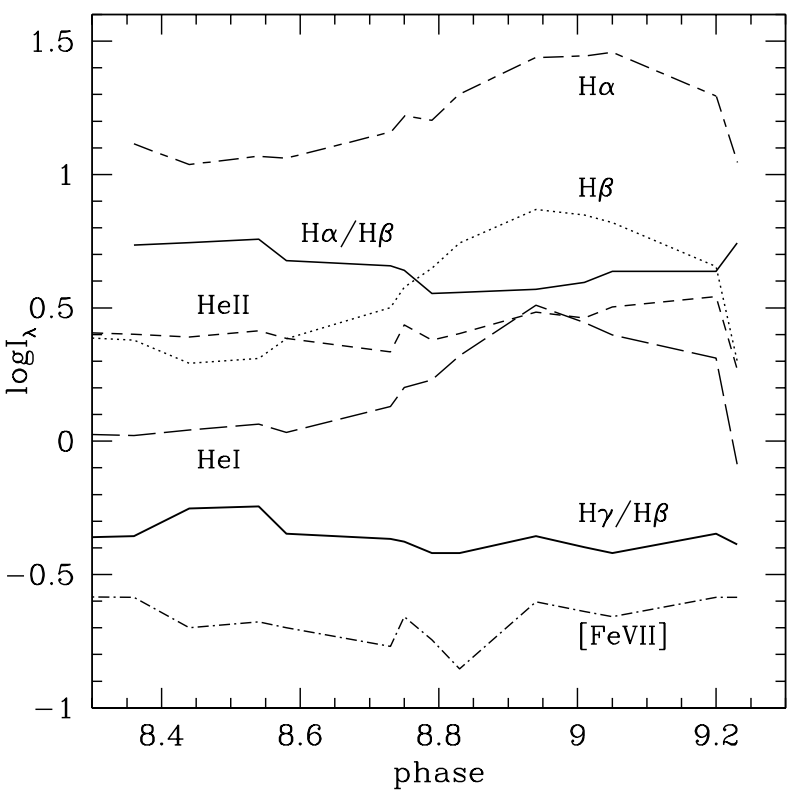

Figure 1. The evolution of some significant line intensities with phase. The lines are HeI 4471, HeII 4686, [FeVII] 6087, H $\beta$, and $\mathrm{H} \alpha$.

(FOS) on board of the Hubble Space Telescope (HST) between late 1993 and late 1996. Low resolution optical spectra with the FAST spectrograph mounted at the F.I. Whipple Observatory 1.5-m telescope are also reported. After an accurate analysis of the data, KPK01 conclude that "if the hot component emits enough high energy photons to account for the forbidden line fluxes, mechanical heating from the colliding winds may explain the high electron temperature. A detailed photoionisation calculation which includes shock excitation would test this proposal." Responding to KPK01, I present further modelling of AG Peg on the basis of their observational data at the latest phases $(\phi=7.39-9.44)$, adopting for the calculations the code SUMA (Viegas \& Contini 1994, Paper I). SUMA calculates the emitted spectrum from the gas, accounting for the coupled effect of shock and photoionisation from an external source. The spectra in the different phases are considered in Sect. 2. The models and the modelling process are presented in Sects. 3 and 4, respectively. Results are discussed in Sect. 5 and concluding remarks appear Sect. 6 .

\section{THE SPECTRA AT DIFFERENT PHASES}

Line fluxes observed by KPK01 were corrected for E(B$\mathrm{V})=0.1$. There are several values for $\mathrm{E}(\mathrm{B}-\mathrm{V})$ in the range 0.08-0.15 obtained by different methods (Tomov, Tomova, \& Raikova 1998, Penston \& Allen 1985, etc.). Some significant line fluxes and line ratios corrected for $\mathrm{E}(\mathrm{B}-\mathrm{V})=0.1$ are given in Fig. 1 as function of the phase. Fig. 1 shows that $\mathrm{H} \alpha / \mathrm{H} \beta$ and $\mathrm{H} \gamma / \mathrm{H} \beta$ at phases $\phi<8.8$ are higher than case $\mathrm{B}$ values. A higher reddening correction of the spectra is not predicted because it would lead to very high values of the far UV line fluxes. Moreover, KPK01 notice that the depth of the $2200 \AA$ absorption feature does not vary with phase and is consistent with $\mathrm{E}(\mathrm{B}-\mathrm{V})=0.1$. Finally, the best fit of the calculated continuum SED to the data of the IR dust bump at early phases (Paper I) corresponds to a relatively low gas-to-dust ratio, $\mathrm{d} / \mathrm{g}=2.10^{-15}$ by number.

We would like to investigate the origin of such high values of $\mathrm{H} \alpha / \mathrm{H} \beta$ and $\mathrm{H} \gamma / \mathrm{H} \beta$. We will refer to radiative transfer effects of HI, to collision excitation of $\mathrm{H}$ lines, and, more specifically, to the effect of the viewing angle of the emitting nebulae.

Recall that densities in the emitting nebulae of symbiotic systems are generally high $\left(>10^{4} \mathrm{~cm}^{-3}\right)$, sufficiently high that some self absorption occurs in the Balmer lines. This leads to a marked strengthening of the $\mathrm{H} \alpha$ line relative to the rest of the Balmer series. In case B, as the optical thickness of Ly $\alpha$ increases, the first effect in the decrement occurs when $\mathrm{H} \beta$ converts into $\mathrm{H} \alpha$ (Cox \& Mathews 1969). Particularly, for large optical thickness of the Ly $\alpha$ line, the increase of the $\mathrm{H} \alpha$ optical thickness leads to an increase of both $\mathrm{H} \alpha / \mathrm{H} \beta$ and $\mathrm{H} \gamma / \mathrm{H} \beta$ (Cox \& Mathews 1969, Fig. 1). In fact, $\mathrm{H} \alpha$ is mostly scattered because any $\mathrm{L} \beta$ photons it forms are quickly absorbed and converted back to $\mathrm{H} \alpha$, while $\mathrm{H} \beta$ is absorbed and converts to $\mathrm{H} \alpha+\mathrm{P} \alpha$.

Moreover, Drake \& Ulrich (1980) claim that $\mathrm{H} \alpha / \mathrm{H} \beta$ ratios greater than 10 can be obtained with optical depths of the order of 100 or more by Balmer self-absorption alone. Particularly, conditions which tend to produce very steep Balmer decrements are found to be large optical depths in $\mathrm{H} \alpha$, low photoionisation rates, and values of the electron density between $10^{8} \mathrm{~cm}^{-3}$ and $10^{12} \mathrm{~cm}^{-3}$ (see also Krolik \& McKee 1978).

In the case of symbiotic systems, collisional processes are very effective, because shock fronts are created by collision of the winds between the stars with velocities $\geqslant 40$ $\mathrm{km} \mathrm{s}^{-1}$. Such velocities correspond to temperatures $\geqslant 20,000$ $\mathrm{K}$ in the postshock region. Higher velocities are actually observed leading to higher temperatures. Collisional excitation increases with temperature and prevails for $\mathrm{T} \geqslant 20,000 \mathrm{~K}$. Collision strengths are $\propto \mathrm{E}_{i j}^{-2} \exp \left(-\mathrm{E}_{i j} / \mathrm{kT}\right.$ ) (Contini \& Aldrovandi 1983) where $\mathrm{E}_{i j}$ is the energy of the level. Radiation from the hot star, on the other hand, cannot heat the gas to $\geqslant 20,000 \mathrm{~K}$. So, high $\mathrm{H} \alpha / \mathrm{H} \beta$ line ratios are predicted in the spectra emitted from shock dominated nebulae.

It was shown in Paper I that the spectra of AG Peg at different epochs are emitted mainly by two nebulae : one in the downstream region of the shock front propagating in reverse towards the hot source and the other downstream of the shock front expanding outwards in the atmosphere of the red giant opposite to the hot source. In particular, the gas is collisionally heated in the outer region of the expanding nebula, while is heated by radiation in the internal region of the same nebula facing the hot source. So, collision dominated spectra will prevail at photometric minimum. Fig. 1 shows that the $\mathrm{H} \alpha / \mathrm{H} \beta$ line ratio has a rough maximum at phase $\sim 8.5$, which could be due to collisional effects. We will come back to this point after modelling the spectra.

When the system evolves through the photometric minimum and the hot source is occulted, the HeII lines that are emitted by a photoionised gas, should strongly decline. On the contrary, Fig. 1 shows that the HeII 4686 flux does not change with phase more than about $22 \%$ throughout the period between phases 8.33 and 9.44. This behavior reveals that nebulae heated and ionised by radiation contribute to 
the spectra near maximum as well as near photometric minimum.

\section{THE MODELS}

SUMA calculates the emitted spectrum from a gas in a plane parallel geometry. The nebula between the stars is ionised and heated by both black body radiation from the WD and the (reverse) shock. Therefore, radiation reaches the very front edge of the shocked nebula. On the other hand, radiation and shock act on opposite edges of the nebula downstream of the expanding shock which propagates in the red giant atmosphere.

The input parameters are : the shock velocity, $\mathrm{V}_{\mathrm{s}}$, the preshock density, $\mathrm{n}_{0}$, the preshock magnetic field, $\mathrm{B}_{0}$, the colour temperature of the hot star, $\mathrm{T}_{*}$, the ionisation parameter, $\mathrm{U}$, the geometrical thickness of the emitting gaseous nebula, D, and the abundance of $\mathrm{He}, \mathrm{C}, \mathrm{N}, \mathrm{O}, \mathrm{Ne}, \mathrm{Mg}, \mathrm{Si}$, $\mathrm{S}, \mathrm{A}$, and Fe relative to $\mathrm{H}$. For all models $\mathrm{T}_{*}=10^{5} \mathrm{~K}$.

We calculated a large grid of models with the following criteria: to fit the most significant line ratios and to be consistent with the modelling reported in Paper I which was for earlier photometric phases.

Three models were selected from the grid, $\mathrm{m} 1, \mathrm{~m} 2$, and $\mathrm{m} 3$. Model $\mathrm{m} 1$ represents the nebula in the downstream region of the shock propagating in reverse toward the WD. The downstream nebula, facing the red giant, is however close to the WD and the photoinising flux from the hot source reaches the shock front edge of the nebula. Model $\mathrm{m} 2$ represents the nebula downstream of the shock propagating outwards the system in the red giant atmosphere opposite to the WD. The downstream nebula toward the WD is ionised and heated by both the shock and radiation from the hot source. Model m3 shows the same shock characteristics of model $\mathrm{m} 2$, but the emitting nebula downstream is not reached by the WD radiation flux. Model m2 is radiation dominated (RD), while $\mathrm{m} 3$ is shock dominated (SD). Model m3 being SD, the emitted spectrum is mainly excited by collision. Recall that at later phases the shock front, merging with the ISM, is disrupted by R-T and K$\mathrm{H}$ instabilities (see Contini \& Formiggini 2001). Therefore, the ionising flux from the hot source will be eventually prevented from reaching the nebula in the downstream region of the shock by intervening matter.

So, in the following, the "reverse shock" will refer to model $\mathrm{m} 1$, the "RD expanding shock" to model $\mathrm{m} 2$, and the "SD expanding shock" to model m3. Models $\mathrm{m} 1$ and $\mathrm{m} 2$ are matter-bound, while $\mathrm{m} 3$ is radiation bound (see Sect. 4.2).

\section{MODELLING THE SPECTRA}

The spectra contain lines in a relatively large range of ionisation levels and in the different wavelength domains. Recall that the distribution of the fractional abundances of the ions downstream (Fig. 2) depends on the input parameters and that the line intensities increase with the volume of gas emitting the corresponding ion and with the square of the density.

The choice of the models depends on line ratios from different ionisation levels and is constrained by the general model of the AG Peg system.

Between $\phi=7.05$ and $\phi=8.07$ the conditions of the reverse shock (between the two stars) could not change sensibly because the reverse shock is most likely a standing shock, so, we adopt input parameters at $\phi=8.07, \mathrm{~V}_{\mathrm{s}}=60$ $\mathrm{km} \mathrm{s}^{-1}$ and $\mathrm{n}_{0}=10^{6} \mathrm{~cm}^{-3}$ similar to those found at $\phi=7.05$, $\mathrm{V}_{\mathrm{s}}=40 \mathrm{~km} \mathrm{~s}^{-1}$ and $\mathrm{n}_{0}=310^{6} \mathrm{~cm}^{-3}$ ( Paper I, Table 4, column 2). In particular, $B_{0}=10^{-3}$ gauss is adopted for $\mathrm{m} 1$, which is suitable for isolated giants (Bohigas et al 1989).

The preshock density of the shock propagating throughout the atmosphere of the red giant in the present modelling $\left(10^{3} \mathrm{~cm}^{-3}\right)$ is lower than that calculated at phases $7.05-7.12$ (Paper I, Table 4 ) by a large factor $(\sim 3000)$. The shock velocity is slightly lower $\left(100 \mathrm{~km} \mathrm{~s}^{-1}\right.$ at $\phi=8.07$ and $150 \mathrm{~km} \mathrm{~s}^{-1}$ at $\left.\phi=7.05\right)$. A lower $\mathrm{n}_{0}$ indicates that the shock has proceeded towards the outskirts of the giant atmosphere. In about 820 days (between $\phi=7.05$ and $\phi=8.07$ ) the shock expanding with a velocity of $\sim 100 \mathrm{~km} \mathrm{~s}^{-1}$ could reach a distance of $\sim 7.10^{14} \mathrm{~cm}$ and is propagating outwards the system, merging with the ISM. Therefore, the preshock density of model $\mathrm{m} 2$ is low and the magnetic field is also reduced to $10^{-4}$ gauss. Both $\mathrm{n}_{0}$ and $\mathrm{B}_{0}$ are, nevertheless, still higher than found in the ISM $\left(1-10 \mathrm{~cm}^{-3}\right.$, and $10^{-5}-$ $10^{-6}$ gauss, respectively).

The relative abundances adopted at $\phi \geqslant 8.07$ are $\mathrm{C} / \mathrm{H}$ $=3.310^{-4}, \mathrm{~N} / \mathrm{H}=5.310^{-4}, \mathrm{O} / \mathrm{H}=9.610^{-4}, \mathrm{Ne} / \mathrm{H}=8.3$ $10^{-5}, \mathrm{Mg} / \mathrm{H}=2.10^{-5}, \mathrm{Si} / \mathrm{H}=3.310^{-5}, \mathrm{~S} / \mathrm{H}=1.610^{-5}$, $\mathrm{A} / \mathrm{H}=6.310^{-6}$, and $\mathrm{Fe} / \mathrm{H}=4.10^{-5}$, as were found at $\phi=7.05-7.12$ (Paper I, Table 4). $\mathrm{Mg} / \mathrm{H}$ is, however, similar to that at $\phi=2.74$ (Paper I, Table 1 ) because at $\phi=7.05$ $\mathrm{Mg}$ lines were not observed and the $\mathrm{Mg} / \mathrm{H}$ abundance ratio could not be determined by modelling the corresponding lines. The adopted relative abundances show that $\mathrm{N} / \mathrm{H}$ and $\mathrm{O} / \mathrm{H}$ ratios are higher than solar. Relatively high values of $\mathrm{N} / \mathrm{H}$ and $\mathrm{O} / \mathrm{H}$ are characteristic of symbiotic systems.

The modelling process of the spectra is roughly described in the following. Recall that model $\mathrm{m} 1$ represents the reverse shock, $\mathrm{m} 2$ the $\mathrm{RD}$ expanding shock, and $\mathrm{m} 3$ the SD expanding shock. The results corresponding to single models are summed up adopting relative weights at each phase and the final results are compared with the observations. The relative weights which lead to the best fit of the observed spectra in the different epochs are related to the configuration of the system and are discussed in the next section.

High ionisation-level lines (e.g. NV, [MgV], [FeVII], etc) increase with the ionisation parameter $\mathrm{U}$ and with $\mathrm{V}_{\mathrm{s}}$. In fact, the temperature downstream behind the shock front is $\propto \mathrm{V}_{\mathrm{s}}{ }^{2}$. NV/CIV ratios calculated by model $\mathrm{m} 1$ with relatively low velocities $\left(\mathrm{V}_{\mathrm{s}}<80 \mathrm{~km} \mathrm{~s}^{-1}\right)$ are lower than observed, therefore, they must be counter-balanced by line ratios higher than observed, calculated by the $\mathrm{m} 2$ model. The NV/CIV ratio calculated by SD models (m3) with $\mathrm{V}_{\mathrm{s}} \sim$ $100 \mathrm{~km} \mathrm{~s}^{-1}$ is very low. So, the only way to obtain a high $\mathrm{NV} / \mathrm{CIV}$ is to adopt a high $\mathrm{U}$ in model $\mathrm{m} 2$. This leads to high HeII/CIV line ratios.

The NV/CIV ratios calculated by a RD model with ionisation and shock acting on opposite edges, are not high enough, if the density is high $\left(10^{6} \mathrm{~cm}^{-3}\right)$. Also, at high densities, the ratio of the intermediate ionisation-level lines (NIII/CIV, SiIII/CIV, and CIII]/CIV) would be higher than 
Table 1

Line ratios to CIV 1550 from selected models

\begin{tabular}{|c|c|c|c|}
\hline line & $\mathrm{m} 1$ & $\mathrm{~m} 2$ & m3 \\
\hline NV 1240 & 0.70 & 3.22 & 0.1 \\
\hline OI 1305 & 0.0 & 0.0 & $1.2(-3)$ \\
\hline SiIV] 1394 & 0.04 & 0.04 & 0.19 \\
\hline SiIV, OIV] 1403 & 0.35 & 1. & 0.23 \\
\hline NIV] 1486 & 0.8 & 1.17 & 0.37 \\
\hline CIV 1550 & 1 & 1 & 1 \\
\hline HeII 1640 & 0.63 & 2.44 & $2.4(-3)$ \\
\hline OIII] 1664 & 0.025 & 0.012 & 0.24 \\
\hline NIV 1719 & $6 .(-3)$ & 0.04 & 5. $(-6)$ \\
\hline NIII] 1750 & 0.08 & 0.04 & 0.85 \\
\hline SiIII] 1892 & 0.006 & 3. $(-3)$ & 0.26 \\
\hline CIII] 1908 & 0.032 & 0.013 & 0.76 \\
\hline CII] 2325 & $5 \cdot(-5)$ & 8. $(-6)$ & 0.35 \\
\hline [MgV] 2783 & 0.024 & 0.15 & 3. $(-5)$ \\
\hline MgII 2793 & $1.3(-4)$ & $2 .(-5)$ & 0.046 \\
\hline$[\mathrm{NeV}] 2976$ & $6.5(-4)$ & 3. $(-3)$ & 6. $(-6)$ \\
\hline HeII 3203 & 0.038 & 0.15 & $1.4(-4)$ \\
\hline$[\mathrm{NeV}] 3426$ & 0.1 & 1.0 & $1.5(-4)$ \\
\hline HI 4340 & 0.04 & 0.145 & 4. $(-3)$ \\
\hline HeI 4471 & $6.2(-5)$ & $7 \cdot(-5)$ & 6. $(-4)$ \\
\hline HeII 4686 & 0.087 & 0.34 & 2. $(-4)$ \\
\hline [FeVII] 6087 & 0.045 & 0.44 & $2.2(-5)$ \\
\hline $\mathrm{CIV} / \mathrm{H} \beta$ & 12.4 & 3.22 & 84.4 \\
\hline $\mathrm{H} \alpha / \mathrm{H} \beta$ & 2.78 & 2.78 & 7. \\
\hline $\mathrm{H} \beta\left(\mathrm{erg} \mathrm{cm}^{-2} \mathrm{~s}^{-1}\right)$ & 237. & 0.003 & $4.2(-4)$ \\
\hline $\mathrm{V}_{\mathrm{S}}\left(\mathrm{km} \mathrm{s}^{-1}\right)$ & 60. & 100. & 100. \\
\hline $\mathrm{n}_{0}\left(\mathrm{~cm}^{-3}\right)$ & $1(6)$ & $1(3)$ & $1(3)$ \\
\hline $\mathrm{B}_{0}\left(10^{-4}\right.$ gauss $)$ & 10. & 1. & 1. \\
\hline $\mathrm{T}_{*}\left(10^{5} \mathrm{~K}\right)$ & 1. & 1. & 1. \\
\hline $\mathrm{U}$ & 0.5 & 5. & - \\
\hline $\mathrm{D}\left(10^{14} \mathrm{~cm}\right)$ & 1.5 & 2. & 2. \\
\hline $\mathrm{s}$ & 0 & 1 & 1 \\
\hline
\end{tabular}

observed by a factor $>10$. High densities are thus rejected for model $\mathrm{m} 2$. This is consistent with the previous discussion (Sect. 3), namely, that the RD expanding shock propagates in a low density medium.

Low ionisation-level line ratios (e.g. CII]/CIV and $\mathrm{MgII} / \mathrm{CIV}$ ) are low for models $\mathrm{m} 1$ and $\mathrm{m} 2$ (Table 1). Low ionisation-level lines are high in SD models, therefore, model m3 must be accounted for. The relatively high CII]/CIV and $\mathrm{MgII} / \mathrm{CIV}$ ratios calculated by $\mathrm{m} 3$ counter-balance in the averaged spectra the low values found by models $\mathrm{m} 1$ and $\mathrm{m} 2$.

A large grid of models is calculated in order to achieve the best fit of all the line ratios.

\subsection{Calculated line ratios}

The line ratios calculated by models $\mathrm{m} 1, \mathrm{~m} 2$, and $\mathrm{m} 3$ are presented in Table 1. The input parameters are given in the bottom of the table. The parameter s, which appears in the last row of Table 1 distinguishes between the two cases: radiation from the star reaches the shocked edge of the emitting nebula $(\mathrm{s}=0)$ and radiation and shock act on opposite edges $(\mathrm{s}=1)$. The calculated absolute $\mathrm{H} \beta$ flux and the $\mathrm{CIV} / \mathrm{H} \beta$ line ratios are also given in order to calculate the flux of the lines. Recall, however, that the spectra are calculated at the nebula but are observed at Earth. The adjusting factor depends on the distance to Earth of AG Peg, as well as on the distances of the nebulae to the WD.

\subsection{Distribution of the different ions throughout the nebulae}

In order to better understand the line ratios calculated by the different models, the distribution of the fractional abundance of some interesting ions as well as of the electron density and temperature downstreams is shown in Fig. 2 for models $\mathrm{m} 1$ (top diagram), m2 (middle diagram), and m3 (bottom diagram). The shock front is on the left; black body radiation reaches the left edge in the top diagram $(\mathrm{m} 1)$ and the right edge in the middle diagram $(\mathrm{m} 2)$. The middle diagram is divided in two equal symmetric parts in order to give equal importance to the shock dominated region (left) and to the radiation dominated region (right). Model m3 (bottom diagram) is shock dominated $(\mathrm{U}=0$.). In all the diagrams the ions are represented by the following symbols. Solid lines : $\mathrm{H}^{+1}$; dotted lines : $\mathrm{He}^{+2}$; short-dashed lines: $\mathrm{N}^{+5}$; dash-dotted lines : $\mathrm{C}^{+4}$; long-dashed lines : $\mathrm{C}^{+3}$; longdash-dotted lines : $\mathrm{C}^{+2}$.

Fig. 2 shows that the temperature throughout the nebulae never decreases below $10^{4} \mathrm{~K}$ downstream of the reverse shock (top diagram). This is due to the coupled effect of a relatively high $\mathrm{U}$ and a relatively small geometrical thickness of the nebula, too small to host a large recombination region.

The profile of the temperature is different in the RD expanding nebula (middle diagram). The gas behind the shock front is heated to $\mathrm{T}>1.510^{5} \mathrm{~K}$ by the shock corresponding to $\mathrm{V}_{\mathrm{s}}=100 \mathrm{~km} \mathrm{~s}^{-1}$. The high ionisation level lines (e.g. $\mathrm{NV}$ ) are strong due to the coupled effects of the relatively high temperature and the strong radiation flux from the hot source. The cooling rate $\left(\propto \mathrm{n}^{2}\right)$ of the gas is sped up by freefree, free-bound, and line emission, therefore a large cool region appears in the left side of the diagram. Notice that $\mathrm{H}$ remains fully ionised and the $\mathrm{N}^{+5} / \mathrm{N}$ fractional abundance is high in the shocked side of the nebula, even if the temperature drops, because they are maintained by the strong radiation flux from the hot source. $\mathrm{C}^{+4}$ prevails throughout the nebula corresponding to the $\mathrm{m} 1$ and $\mathrm{m} 2$ models owing to the high $\mathrm{U}$.

The shock dominated model (m3) shows different conditions, characteristic of SD models. A relative large region is dominated by the $\mathrm{C}^{+2}$ ion. The absolute flux of the $\mathrm{H} \beta$ line is low because the emitting region throughout the nebula is relatively small.

Interestingly, $\mathrm{H} \alpha / \mathrm{H} \beta$ is higher in the postshock region (left side of the middle diagram) of model $\mathrm{m} 2$ than in the region reached by the radiation from the hot source (right side) and is definitively high throughout all the nebula represented by model $\mathrm{m} 3$.

\section{RESULTS}

The best modelling of AG Peg should, indeed, imply a huge number of models in order to represent the complex nature of symbiotic systems (see Nussbaumer 2000). The models, 

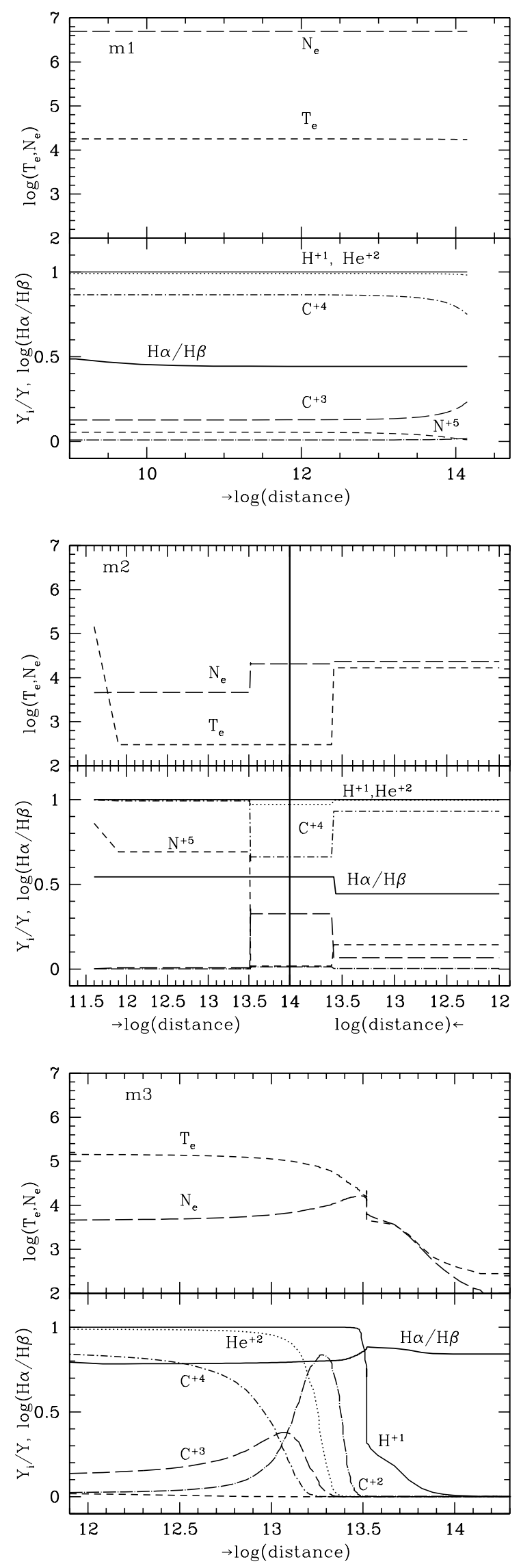

Figure 2. see text

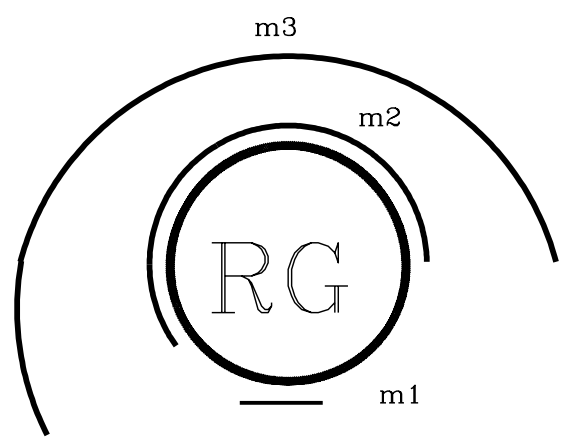

(WD)

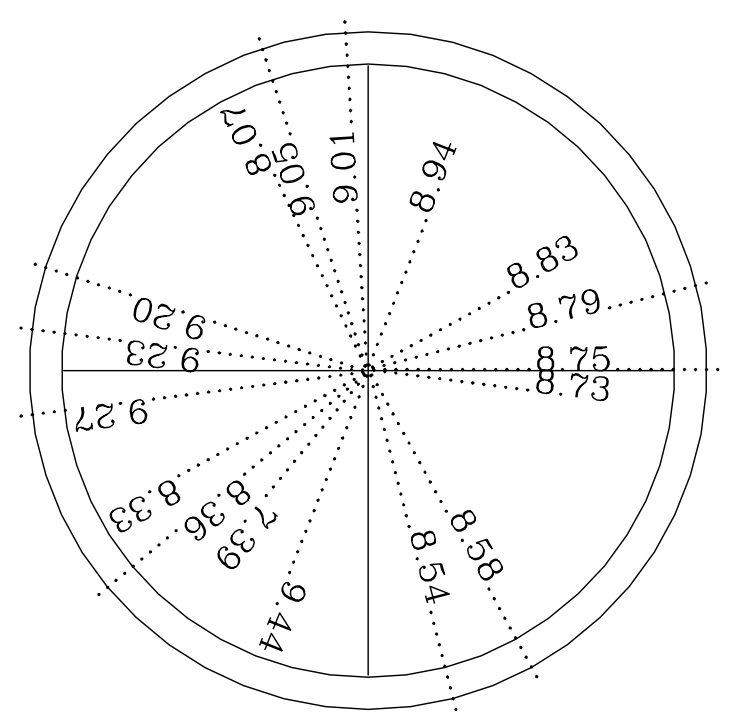

Figure 3. Top panel : A schematic representation of AG Peg system. Bottom panel : The orientation of the binary system axis at different phases.

however, are schematized to three main types representing the three emitting nebulae described in the previous sections; therefore, the results show only a consistent approach.

\subsection{Comparison of calculated with observed line ratios}

The calculated spectra at each phase are compared with observations in Table 2. Each spectrum results from the weighted sum of the three models. The relative weights of the models are shown in the last rows of Table 2 . The weights are very different for different models because they depend on the emitting area of the corresponding nebula. 
Table 2

The comparison of calculated and observed line ratios to CIV 1550

\begin{tabular}{|c|c|c|c|c|c|c|c|c|}
\hline \multirow[b]{2}{*}{ line } & \multicolumn{2}{|c|}{$\phi=8.07$} & \multicolumn{2}{|c|}{$\phi=8.33$} & \multicolumn{2}{|c|}{$\phi=9.27$} & \multicolumn{2}{|c|}{$\phi=9.44$} \\
\hline & obs & calc & obs & calc & obs & calc & obs & calc \\
\hline NV 1240 & 0.90 & 0.8 & 1.16 & 1.3 & 1.04 & 1.18 & 1.19 & 2.39 \\
\hline OI 1305 & 0.06 & 0.0001 & 0.031 & 0.0002 & 0.044 & 0.0001 & 0.03 & 0.0003 \\
\hline SiIV] 1394 & 0.06 & 0.05 & 0.05 & 0.06 & 0.054 & 0.057 & 0.04 & 0.08 \\
\hline SiIV, OIV] 1403 & 0.22 & 0.35 & 0.56 & 0.40 & 0.317 & 0.38 & 0.50 & 0.47 \\
\hline NIV] 1486 & 0.21 & 0.8 & 0.27 & 0.84 & 0.262 & 0.83 & 0.47 & 0.98 \\
\hline CIV 1550 & 1 & 1. & 1 & 1 & 1. & 1 & 1 & 1. \\
\hline HeII 1640 & 0.61 & 0.67 & 1.33 & 1.1 & 1.276 & 1.0 & 2.58 & 1.90 \\
\hline OIII] 1664 & 0.18 & 0.03 & 0.25 & 0.05 & 0.227 & 0.05 & 0.41 & 0.07 \\
\hline NIV 1719 & 0.05 & 0.01 & 0.10 & 0.014 & 0.085 & 0.012 & 0.12 & 0.03 \\
\hline NIII] 1750 & 0.05 & 0.057 & 0.09 & 0.10 & 0.084 & 0.085 & 0.14 & 0.13 \\
\hline SiIII] 1892 & 0.05 & 0.02 & 0.06 & 0.05 & 0.056 & 0.04 & 0.08 & 0.07 \\
\hline CIII] 1908 & 0.05 & 0.06 & 0.10 & 0.14 & 0.093 & 0.11 & 0.17 & 0.20 \\
\hline CII] 2325 & 0.01 & 0.016 & 0.007 & 0.06 & 0.01 & 0.04 & 0.04 & 0.09 \\
\hline$[\mathrm{MgV}] 2783$ & 0.012 & 0.027 & 0.038 & 0.05 & 0.038 & 0.048 & 0.08 & 0.11 \\
\hline MgII 2793 & 0.02 & 0.002 & 0.024 & 0.01 & 0.022 & 0.013 & 0.03 & 0.014 \\
\hline$[\mathrm{NeV}] 2976$ & 0.004 & 0.001 & 0.006 & 0.0012 & 0.005 & 0.001 & 0.01 & 0.002 \\
\hline HeII 3203 & 0.04 & 0.04 & 0.103 & 0.07 & 0.01 & 0.05 & 0.21 & 0.11 \\
\hline$[\mathrm{NeV}] 3426$ & 0.022 & 0.13 & 0.073 & 0.35 & 0.023 & 0.30 & 0.15 & 0.74 \\
\hline HI 4340 & 0.056 & 0.04 & 0.07 & 0.06 & 0.048 & 0.058 & 0.113 & 0.110 \\
\hline HeI 4471 & 0.004 & 0.0001 & 0.007 & 0.0001 & 0.005 & 0.0001 & 0.010 & 0.0002 \\
\hline HeII 4686 & 0.06 & 0.08 & 0.159 & 0.14 & 0.131 & 0.129 & 0.273 & 0.250 \\
\hline$[\mathrm{FeVII}] 6087^{1}$ & - & 0.06 & 0.019 & 0.15 & 0.012 & 0.128 & - & - \\
\hline $\mathrm{w}(\mathrm{m} 1)$ & - & $1.7(-3)$ & - & $1.3(-4)$ & - & 2. $(-4)$ & - & 0.0 \\
\hline $\mathrm{w}(\mathrm{m} 2)$ & - & 20 & - & 20. & - & 20. & - & 20. \\
\hline $\mathrm{w}(\mathrm{m} 3)$ & - & 6.67 & - & 3.0 & - & 3.0 & - & 2. \\
\hline
\end{tabular}

${ }^{1}[\mathrm{FeVII}]$ flux from the FAST spectra at $\phi=8.36$ and $\phi=9.27$

A very schematic representation of the system and the angles of view at the different phases are given in Fig. 3 (top panel and bottom panel, respectively).

At $\phi=8.07$ we see almost all of the shock fronts, internal and external to the system (Fig. 3). The distance of the reverse shock from the white dwarf, $\mathrm{r}_{1}$, is of the order of the binary separation (in Paper I it is found that the front is very close to the red giant surface), so, we adopt $r_{1}=510^{13}$ $\mathrm{cm}$; the radius of the $\mathrm{RD}$ expanding front is $\mathrm{r}_{2}(\phi=8.07)=$ $\mathrm{r}_{2}(\phi=7.05)+\Delta \mathrm{r}$, where $\Delta \mathrm{r}$ is the distance covered by the shock front between phases 8.07 and 7.05. Then, $\mathrm{r}_{2}=3.2$ $10^{15} \mathrm{~cm}$ (Paper I) $+710^{14} \mathrm{~cm}$ (Sect. 4) is $\sim 410^{15} \mathrm{~cm}$.

Actually, $\left(\mathrm{r}_{2} / \mathrm{r}_{1}\right)^{2} \sim 6400$ at $\phi=8.07$ is lower than $\mathrm{w}(\mathrm{m} 2) / \mathrm{w}(\mathrm{m} 1)=1.210^{4}$ by a factor $<2$. The ratios of the weights which appear in Table 2 are determined by the best fit of the calculated to the observed data. The discrepancy by a factor of $<2$ indicates that the radiation emitted by the reverse shock is slightly absorbed by the outer atmosphere of the red giant. At later phases, $\phi=8.33$ and $\phi=9.27$, the radiation intersects the red giant at angles that depend on the phase angle, and the path length throughout the red giant, $\mathrm{l}=2 \mathrm{r}_{R G} \cos \alpha\left(\mathrm{r}_{R G}\right.$ is the radius of the red giant and $\alpha$ is the angle between the binary system axis and the viewing direction from Earth), is higher at $\phi=8.33$ than at $\phi=9.27$. So, at $\phi=9.27$ the radiation from the reverse shock is less absorbed than at $\phi=8.33$, in agreement with the trend of the $\mathrm{w}(\mathrm{m} 2) / \mathrm{w}(\mathrm{m} 1)$ ratio that is $10^{5}$ at $\phi=9.27$ and is 1.5
$10^{5}$ at $\phi=8.33$. The trend is clearly depending on the phase angle, however, the trend does not depend only on the angle because the radius of the expanding shock is not symmetric around the system (see Nussbaumer 2000, Fig. 1).

Regarding the SD shock, the ratio of the relative weights $\mathrm{w}(\mathrm{m} 3) / \mathrm{w}(\mathrm{m} 2)$ does not follow any specific trend, because it depends on fragmentation of matter and on the filling factor. Also those ratios were chosen by the best fit to the data.

Considering that the AG Peg system is very complex, we have focused on the most significant lines, e.g. NV 1240, CIV 1550, HeII 1640, CIII] 1908, CII] 2325, etc. in the UV, and HeII 4686 in the optical range. NIV] 1486 is overestimated by the calculations. OI 1305, HeI 4471 and MgII are underestimated by a large factor. The contribution from a model corresponding to a large low temperature region could improve this result. [NeV] 3426/ CIV line ratio is overpredicted. Notice, however, that this ratio refers to lines emitted from one or two different nebulae, indicating that more model components should be considered. The same is valid for the [FeVII] 6087 / CIV line ratio. In this case, however, some iron could be locked into dust grains.

A slightly lower $\mathrm{N} / \mathrm{H}$ could improve the fit of the $\mathrm{NV}$ 1240 and NIV] 1486 line ratios to CIV, particularly at phase 9.44. N/H approaching to the ISM value, and the relatively low $\mathrm{n}_{0}$ and $\mathrm{B}_{0}$ adopted for models $\mathrm{m} 2$ and $\mathrm{m} 3$, confirm that the merging process is taking place.

In the averaged spectra we found $\mathrm{H} \alpha / \mathrm{H} \beta<3$, lower than 
the observed line ratios, because the weights of $\mathrm{m} 1$ and $\mathrm{m} 2$ models are relatively high. A possible explaination is given by Shore (1992) who claims that in a moving medium with an internal velocity gradient, the optical depth of the line $\Delta \tau$ $\sim \Delta \mathrm{v} /(\mathrm{dv} / \mathrm{dl}) . \Delta \mathrm{v}=\Delta \nu_{D}$, where $\Delta \nu_{D}$ is the Doppler width of a line with rest frequency $\nu_{0}$. The reduction of the velocity gradient causes a rapid increase in the line opacity, even if the density has dropped enough as to make the equivalent static line profile completely optically thin. Indeed, a velocity gradient is predicted in the outer atmosphere of the red giant. Adopting $\mathrm{V}_{\mathrm{s}}=150 \mathrm{~km} \mathrm{~s}^{-1}$ at $\phi=7.12$ (Contini 1997), $\mathrm{V}_{\mathrm{s}}=100 \mathrm{~km} \mathrm{~s}^{-1}$ at the latest epochs, and $\mathrm{dl}=10^{14}$ $\mathrm{cm}, \Delta \tau$ should increase by a factor $>10^{7}$, leading to higher $\mathrm{H} \alpha / \mathrm{H} \beta$ (see Sect. 2).

We can now compare the luminosities of the lines calculated by the models with those observed by KPK01. Considering, for instance $[\mathrm{NeV}] 3426$ at $\phi=8.07$, the calculated luminosity is $\sim 310^{30} \mathrm{erg} \mathrm{s}^{-1}$ while the observed one (KPK01, Table 4) is $410^{31} \mathrm{erg} \mathrm{s}^{-1}$ adopting a distance to Earth, $d=800$ pc. This line is mainly emitted from the nebula corresponding to model $\mathrm{m} 1$. A better fit to the observed luminosity could be obtained adopting $\mathrm{n}_{0}=310^{6} \mathrm{~cm}^{-3}$ instead of $10^{6} \mathrm{~cm}^{-3}$ for $\mathrm{m} 1$. The preshock density used at $\phi$ $=7.05$ (Paper I, Table 4) was $310^{6} \mathrm{~cm}^{-3}$.

The distance of the SD expanding nebula from the center of the binary system, $\mathrm{r}_{3}$, at $\phi=9.44$ is obtained comparing the luminosities of calculated and observed CII] lines. From CII $\left.]_{o b s} \times \mathrm{d}^{2}=\mathrm{CII}\right]_{m 3} \times \mathrm{r}_{3}^{2}, \mathrm{r}_{3}$ results $\sim 10^{16} \mathrm{~cm}$. The CII] line is considered because it is emitted mainly from the outer SD nebula at photometric minimum. The distance is larger by a factor $>2$ than the distance of the RD expanding nebula corresponding to model $\mathrm{m} 2$. Even if the RD and SD expanding shocks are at different distances from the system center, they may belong to the same wave. In fact, the "fingers" created by R-T and K-H instabilities at the shock front can be elongated up to a large fraction of the shock front radius (see Contini \& Formiggini 2001).

In summary, the interpretation of the data by a relatively small number of models is sound, even if approximated. Anyway, some interesting results follow.

\subsection{Relative contribution of the different nebulae to the spectra}

The relative contribution of the emitting nebulae to each line is shown in Table 3. The weight of models $\mathrm{m} 2$ and $\mathrm{m} 3$, representing the nebulae propagating outwards in the atmosphere of the red giant, relative to the weight of the nebula corresponding to the reverse shock between the stars increases with the angle determined by the phase fraction $(.07, .27, .33$, and .44), indicating that the external nebula is not present around the whole system, but only in the region opposite to the WD.

The nebula downstream of the reverse shock dominates at $\phi=8.07$ except for a few lines (SiIII], CIII], CII], and $\mathrm{MgII}$ ). A large contribution to these lines comes from the SD expanding shock. The RD expanding shock contributes partly to NIV, [NeV], and to [FeVII].

The relative contribution of the three nebulae to the lines are similar at $\phi=8.33$ and at $\phi=9.27$, the reverse shock being less dominant and indicating that the relative contributions of the nebulae depend on the viewing angle of the system rather than on the phase. At both phases 8.33 and 9.27 the $\mathrm{RD}$ expanding shock is dominating for most of the lines (NV, HeII 1640, NIV, [MgV], [NeV], HeII 3203, [NeV] 3426, HI 4340, HeII 4686, [FeVII], and $\mathrm{H} \alpha$ ). The SD expanding shock dominates the low ionisation level lines.

At $\phi=9.44$ the contribution from the reverse shock becomes negligible because the nebula between the stars is occulted by the red giant. The high ionisation level lines are produced mainly by the RD expanding shock and low ionisation level lines come from the SD nebula.

The relative contribution of the nebula between the stars to the HeII 1640 line decreases monotonically with phase fractions $.07, .27, .33$, and .44 On the other hand, the relative contributions of $\mathrm{m} 2$ to NV 1240 are $14.8 \%, 61.4 \%$, $68.75 \%$, and $99.0 \%$, at the same angles, respectively.

Concluding, the evolution of the spectra is more likely related to the viewing angle of the system, not particularly to the phase between $\phi=8.07$ and $\phi=9.44$. We do not have data relative to viewing angles higher than 0.44 , which could hopefully strengthen the results.

\subsection{Evolution of the line fluxes with phase}

It is now possible to understand the trend of the lines shown in Fig. 1. Table 3 shows that the contribution from the SD shocked nebula $(\mathrm{m} 3)$ to $\mathrm{H} \alpha$ is only a few \% , therefore, the calculated $\mathrm{H} \alpha / \mathrm{H} \beta$ line ratio is likely to be $\sim 3$ throughout the whole period between phase 8.07 and 9.44 . On the other hand, the observed value is $\geqslant 5$ towards photometric minimum. This high value may be explained considering that the $\mathrm{RD}$ expanding shock is represented by a composite model which accounts for both the photoionisation by the WD and the shock. The side of the emitting nebula facing the observer at photometric minimum is shock dominated (Fig. 2, middle diagram). So, in this phase we see mainly $\mathrm{H} \alpha$ and $\mathrm{H} \beta$ fluxes from this side of the nebula, which are produced by collisional ionisation. Moreover, the optical depth in the radiation dominated region is high (see Sect. 4.1) leading to high $\mathrm{H} \alpha / \mathrm{H} \beta$.

HeII 4686 and [FeVII] do not show a periodic change with phase because a large fraction of these lines is emitted by the nebula downstream of the RD expanding shock. So, the decrease of the reverse shock contribution at phases approaching the photometric minimum is compensated by the large contribution from the RD expanding shock. These lines show, therefore, less impressive variability up to $\phi \geqslant$ 9.2. At $\phi=9.27$ the calculated $\mathrm{F}_{\lambda 4686}$ is $0.11 \mathrm{erg} \mathrm{cm}^{-2} \mathrm{~s}^{-1}$, while at $\phi=9.44 \mathrm{~F}_{\lambda 4686}=0.06 \mathrm{erg} \mathrm{cm}^{-2} \mathrm{~s}^{-1}$. The decrease of the calculated flux between these two phases is in agreement with the observed decrease shown in Fig. 1 after $\phi=$ 9.27 .

HeI 4471 is emitted mainly by the SD expanding shock (m3) and the flux is very low, $\mathrm{F}_{\lambda 4471}=210^{-5} \mathrm{erg} \mathrm{cm}^{-2} \mathrm{~s}^{-1}$. Therefore, this line flux shows a maximum in Fig. 1 when the system is near maximum. The same is valid for $\mathrm{H} \alpha$ and $\mathrm{H} \beta$. Notice, however that HeI 4471/CIV line ratio is underpredicted by the models.

All the lines show an asymmetric behaviour before and after maximum. This is explained by the fact that the emitting nebulae are not symmetric relative to the axis joining the center of the WD with the center of the red giant. In the hydrodynamical picture of symbiotic systems presented by 
Table 3

Relative contributions (in \%) to the line intensities

\begin{tabular}{|c|c|c|c|c|c|c|c|c|c|c|c|c|}
\hline \multirow[b]{2}{*}{ line } & \multicolumn{3}{|c|}{$\phi=8.07$} & \multicolumn{3}{|c|}{$\phi=8.33$} & \multicolumn{3}{|c|}{$\phi=9.27$} & \multicolumn{3}{|c|}{$\phi=9.44$} \\
\hline & $\mathrm{m} 1$ & $\mathrm{~m} 2$ & $\mathrm{~m} 3$ & $\mathrm{~m} 1$ & $\mathrm{~m} 2$ & $\mathrm{~m} 3$ & $\mathrm{~m} 1$ & $\mathrm{~m} 2$ & $\mathrm{~m} 3$ & $\mathrm{~m} 1$ & $\mathrm{~m} 2$ & $\mathrm{~m} 3$ \\
\hline NV 1240 & 84.7 & 14.78 & 0.54 & 30.11 & 68.75 & 1.13 & 37.60 & 61.38 & 1.02 & 0.0 & 99.0 & 0.99 \\
\hline SiIV] 1394 & 78.90 & 3.68 & 17.40 & 34.4 & 20.98 & 44.61 & 44.66 & 17.7 & 37.64 & 0.0 & 43.69 & 56.31 \\
\hline SiIV,OIV] 1403 & 91.35 & 5.39 & 3.25 & 50.45 & 38.96 & 10.59 & 61.04 & 30.64 & 8.32 & 0.0 & 85.86 & 14.14 \\
\hline NIV] 1486 & 92.9 & 5.25 & 1.81 & 53.94 & 39.88 & 6.19 & 64.3 & 30.9 & 4.79 & 0.0 & 91.41 & 8.59 \\
\hline CIV 1550 & 92.27 & 3.57 & 4.16 & 56.47 & 28.56 & 14.97 & 66.62 & 21.9 & 11.48 & 0.0 & 75.89 & 24.11 \\
\hline HeII 1640 & 86.94 & 13.05 & 0.014 & 33.74 & 66.23 & 0.032 & 43.92 & 56.05 & 0.026 & 0.0 & 99.97 & 0.029 \\
\hline OIII] 1664 & 67.78 & 1.35 & 30.87 & 25.39 & 6.60 & 68.01 & 34.36 & 5.81 & 59.83 & 0.0 & 13.81 & 86.19 \\
\hline NIV 1719 & 79.63 & 20.36 & 0.003 & 23.02 & 76.98 & 0.0051 & 31.51 & 68.49 & 0.0045 & 0.0 & 100. & 0.0 \\
\hline NIII] 1750 & 64.84 & 1.35 & 33.8 & 23.04 & 6.29 & 70.66 & 31.54 & 5.60 & 62.86 & 0.0 & 12.81 & 87.19 \\
\hline SiIII] 1892 & 30.21 & 0.64 & 69.14 & 6.78 & 1.89 & 91.32 & 10.0 & 1.82 & 88.11 & 0.0 & 3.30 & 96.70 \\
\hline CIII] 1908 & 47.39 & 0.81 & 51.74 & 13.07 & 2.93 & 84.0 & 18.78 & 2.74 & 78.48 & 0.0 & 5.44 & 94.55 \\
\hline CII] 2325 & 0.27 & 0.002 & 99.73 & 0.05 & 0.004 & 99.95 & 0.071 & 0.004 & 99.93 & 0.0 & 0.0074 & 99.99 \\
\hline [MgV] 2783 & 81.05 & 18.94 & 0.0035 & 24.65 & 75.34 & 0.006 & 33.48 & 66.50 & 0.0056 & 0.0 & 99.99 & 0.0052 \\
\hline MgII 2793 & 4.09 & 0.033 & 94.88 & 0.90 & 0.077 & 99.02 & 1.38 & 0.077 & 98.54 & 0.0 & 0.128 & 99.87 \\
\hline$[\mathrm{NeV}] 2976$ & 85.60 & 14.37 & 0.032 & 31.28 & 68.65 & 0.069 & 41.19 & 58.75 & 0.058 & 0.0 & 99.94 & 0.06 \\
\hline HeII 3203 & 87.0 & 12.98 & 0.013 & 33.88 & 66.09 & 0.031 & 44.08 & 55.89 & 0.026 & 0.0 & 99.97 & 0.028 \\
\hline$[\mathrm{NeV}] 3426$ & 73.3 & 26.66 & 0.0043 & 17.38 & 82.61 & 0.006 & 24.46 & 75.54 & 0.0056 & 0.0 & 100. & 0.0 \\
\hline HI 4340 & 86.7 & 12.93 & 0.37 & 33.61 & 65.56 & 0.836 & 43.78 & 55.5 & 0.71 & 0.0 & 99.23 & 0.77 \\
\hline HeI 4471 & 69.80 & 2.99 & 27.2 & 25.95 & 14.56 & 59.49 & 35.03 & 12.77 & 52.2 & 0.0 & 28.76 & 71.24 \\
\hline HeII 4686 & 85.94 & 14.09 & 0.009 & 31.78 & 68.20 & 0.012 & 41.75 & 58.23 & 0.017 & 0.0 & 99.98 & 0.0177 \\
\hline $\mathrm{H} \beta 4861$ & 100. & 0.000 & 0.0 & 100.0 & 0.0 & 0.0 & 100.0 & 0.0 & 0.0 & 0.0 & 99.99 & 0.007 \\
\hline [FeVII] 6087 & 72.84 & 27.16 & 0.0015 & 17.02 & 82.98 & 0.002 & 23.98 & 76.0 & 0.002 & 0.0 & 100. & 0.0 \\
\hline $\mathrm{H} \alpha 6563$ & 85.77 & 12.79 & 1.43 & 32.8 & 63.98 & 3.22 & 42.89 & 54.38 & 2.74 & 0.0 & 97.04 & 2.96 \\
\hline
\end{tabular}

Nussbaumer (2000, Fig. 1 left) the wake is, in fact, strongly asymmetric, so that the emitting nebulae could partly disappear in some location at angles corresponding to high phase fractions.

\section{CONCLUDING REMARKS}

The analysis of the spectra from the HST and from the FAST spectrograph at the Whipple Observatory reported by Kenyon et al (2001) at different phases leads to new results about the configuration of AG Peg. The FAST spectra contain both $\mathrm{H} \alpha$ and $\mathrm{H} \beta$ lines, providing the tool for modelling the system at different phases. Interestingly, the trend of the periodical variability of $\mathrm{H} \alpha / \mathrm{H} \beta$, as well as the trend of the most significant lines indicate that different nebulae contribute to the final spectra. In particular, the high $\mathrm{H} \alpha / \mathrm{H} \beta$ line ratios $(\sim 5)$ suggest that shock dominated models must be accounted for. Moreover, the trend of the HeII 4686 line variability with phase indicates that radiation dominated nebulae are strongly contributing to the spectra also near the photometric minimum.

Consistent modelling by the code SUMA, which accounts for the coupled effect of photoionisation from the hot star and shocks, shows that at least three nebulae provide the spectral components : 1) the nebula between the stars, downstream of the shock created by head-on collision of the winds, which propagates in reverse towards the WD, 2) the nebula downstream of the head-on-back shock expanding in the outskirts of the red giant atmosphere and reached by the photoionising flux from the WD, and 3) the nebula down- stream of the same shock front which is shock dominated, i.e. it is not reached by the photoionising radiation flux. In fact, the matter in this region is very clumpy because the shock front is disrupted by $\mathrm{R}-\mathrm{T}$ instability, and radiation from the $\mathrm{WD}$ is prevented from reaching the region by the intervening clumps in some locations.

The fit of calculated to observed line ratios is acceptable enough to justify the choice of the models, so that the relative contribution of the different nebulae to the final spectra at different phases can be calculated for all the lines. It is found that the contribution of the nebula between the stars prevails for most of the lines near maximum $(\phi=8.07)$, declining towards higher phases and even disappearing at phase 9.44. Low ionisation level lines (e.g. CII] and MgII) are mainly emitted from the SD expanding nebula. The evolution of the spectra between phases 7.34 and 9.44 is more likely related with the viewing angle of the system, and not to the temporal evolution of the system.

Models $\mathrm{m} 2$ and $\mathrm{m} 3$ which represent the expanding shock are characterized by a relatively low preshock density and magnetic field, much lower than found by modelling AG Peg at $\phi \leqslant 7.12$ (Paper I). This suggests that the shock, expanding in the red giant atmosphere opposite to the WD, has already reached the outskirts of the system and is merging with the ISM. At $\phi=9.44$ the expanding shock has swept up a large portion of matter with relatively low density. The line and continuum fluxes emitted from downstream the shock are $\propto \mathrm{n}^{2}$ and, therefore, decrease as more IS matter is swept up, leading to the slow decline of AG Peg continuum and line fluxes observed by KPK01. 


\section{AKNOWLEDGMENTS}

I am very grateful to the referee for many enlightening suggestions and precious remarks, and to D. Prialnik and S. Beck for reading the manuscript.

\section{REFERENCES}

Bohigas, J., Echevarria, J., Diego, F., \& Sarmiento, J.A. 1989, MNRAS, 238, 1395

Contini, M. 1997, ApJ, 483, 898 (Paper I)

Contini, M. \& Formiggini, L. 2001, A\&A, 375, 579

Cox, D. P. \& Mathews, W.G. 1969, ApJ, 155, 859

Drake, S.A. \& Ulrich, R.K. 1980 ApJS, 42, 351

Kenyon et al. 1993, AJ, 106, 1573

Kenyon, S.J., Proga, D., \& Keyes, C.D. 2001, AJ, 122, 349

Krolik, J.H. \& McKee, C.F. 1987, ApJS, 37, 459

Nussbaumer, H. 2000 in "Thermal and Ionization Aspects of Flows from Hot Stars: Observations and Theory, ASP Conference Series, Vol. 204, Lamers and Sapar eds., p. 317 Osterbrock, D.E. 1989 in "Astrophysics of Gaseous Nebulae and Active Galactic Nuclei", (Mill Valley: University Science Books

Penston, M.V. \& Allen, D.A. 1985 MNRAS, 212, 939

Shore, S.N. 1992 in "An Introduction to Astrophysical Hydrodynamics, Academic Press Inc.

Tomov, N.A., Tomova, M.T., \& Raikova, D.V. 1998, A\&AS, 129, 479

Viegas, S.M. \& Contini, M. 1994, ApJ, 428, 113

This paper has been typeset from a $\mathrm{TEX}_{\mathrm{E}} \mathrm{IAT} \mathrm{T} \mathrm{X}$ file prepared by the author. 